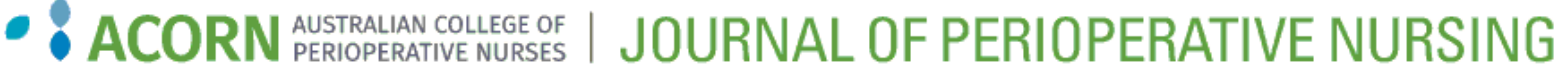

\title{
Can the role of the nurse as surgical assistant add value in the Australian public health care sector?
}

Follow this and additional works at: https://www.journal.acorn.org.au/jpn

Part of the Medical Education Commons, and the Perioperative, Operating Room and Surgical Nursing Commons

(c) (7)

This work is licensed under a Creative Commons Attribution 4.0 License.

\section{Recommended Citation}

Hains, Toni; Navaratnam, Anojan Dr; O'Brien, Andrew; Walsh, Karen; Neilsen, Jane; and Firth, Susan (2021) "Can the role of the nurse as surgical assistant add value in the Australian public health care sector?," Journal of Perioperative Nursing: Vol. 34 : Iss. 1 , Article 2.

Available at: https://doi.org/10.26550/2209-1092.1108

https://www.journal.acorn.org.au/jpn/vol34/iss1/2

This Discussion paper is brought to you for free and open access by Journal of Perioperative Nursing. It has been accepted for inclusion in Journal of Perioperative Nursing by an authorized editor of Journal of Perioperative Nursing. 


\section{Authors}

Toni G. Hains

MClinSc(PNSA), MNPractSt, PhD Scholar, (University of Queensland), NP, FACORN Royal Brisbane and Women's Hospital

Dr Ano Navaratnam

MBBS BSci(MedSci), FRACS

Royal Brisbane and Women's Hospital

Andrew O'Brien

MHealthSci(Ed), GradCertClinNursing(Ed), BNursing, RN

Royal Brisbane and Women's Hospital

Karen Walsh

MNPractSt, BNursing, NP

Royal Brisbane and Women's Hospital

Jane Nielsen

BNursing, RN

Royal Brisbane and Women's Hospital

Susan Firth

MNursingEd, RN

Royal Brisbane and Women's Hospital

\section{Can the role of the nurse as surgical assistant add value in the Australian public health care sector?}

\section{Introduction}

Registered nurses (RNs) and subsequently nurse practitioners (NP) have practiced the role of surgical assistant in the Australian health care system for more than 30 years'. The Australian College of Perioperative Nurses (ACORN) has a statement outlining the responsibilities of nurses undertaking the role? Australian research corroborates international research that non-medical surgical assistants (i.e. surgical assistants who are not medical doctors) have equivalent patient outcomes to their medical counterparts ${ }^{3}$. While the majority of nurses as surgical assistants work in the private sector in the Australian health care system ${ }^{4}$, the role is underutilised in the Australian public health care sector. There is an opportunity for nurses as surgical assistants to add value in the public health care sector. The public sector nurse surgical assistant model is advantageous in surgical specialties where the surgical assistant requires highly specialised skills. These skills often require a protracted learning curve which is not suited to transient junior medical personnel. One specialty particularly suited to nurses as surgical assistants is in robotic-assisted surgery.

\section{Discussion}

\section{The da Vinci robot}

The term 'robot' is open to interpretation depending on the source of the definition, with some claiming a robot must possess artificial intelligence allowing for independent operation ${ }^{5,6}$. The da Vinci robot is a computer-assisted mechanical device, and while it has revolutionised laparoscopic surgery, it operates on a master-slave platform $^{7-9}$. Despite this, the da Vinci system is globally referred to as a robot $^{6}$.

The literature reports advantages of robotic surgery as resulting in a shorter length of stay, less postoperative pain, a quicker return to normal activity and reduced trauma to the body ${ }^{9-11}$. The telescope of the da Vinci robot has two highresolution fibre optic cameras (called 'eyes') which give the operator a three-dimensional view of the surgical site. The use of the da Vinci robot to perform surgical procedures offers the primary surgeon enhanced dexterity and visualisation, particularly in cavities such as the pelvis; however, in order to make use of these benefits the surgeon must be physically separated from the patient ${ }^{12,13}$ with the surgeon carrying out their surgical manoeuvres unscrubbed and in a console remote from the patient. In the literature the primary surgeon is referred to as the console surgeon. For some surgeons, being physically separated from the patient has been a factor in limiting the utilisation of the robot ${ }^{14}$ with surgeons of many specialties expressing apprehension concerning the experience of their tableside assistant 8,12-14 $^{\text {. The table-side }}$ assistant in robotic-assisted surgery acts as a conduit between the 
surgeon and the patient. The person undertaking the role of table-side assistant can vary from a consultant surgeon or other medically qualified personnel, to a mid-level provider such as a physician's assistant, nurse practitioner or perioperative nurse surgical assistant. In training hospitals the table-side assistant has traditionally been a surgical resident, registrar or trainee ${ }^{14,15}$.

Tasks of the surgical assistant may include initial insufflation of the operative cavity, trocar placements, robot docking and undocking, specimen extraction, and drainage and closure of the operative cavity. All da Vinci robot-assisted procedures require the tableside assistant to perform robotic instrument exchanges, laparoscopic instrument manipulation, haemostatic instrument application, lavage and aspiration ${ }^{8,16}$.

\section{The Royal Brisbane and Women's Hospital}

Metro North Hospital and Health Service (MNHHS) is the largest health service in Australia and has a three-year robotic surgery plan spanning 2018 to 2021. The scope of this plan is to offer robotic-assisted procedures in the specialties of general surgery; urology; gynaecology; colorectal; hepato-biliary; ear, nose and throat; head and neck; thoracic; orthopaedics; neurosurgery and ophthalmology ${ }^{10}$. Not all of these specialties will utilise the da Vinci system.

The Royal Brisbane and Women's Hospital (RBWH) was the first public hospital in Australia to commence government-funded robotic surgery, in 2008. RBWH purchased their first da Vinci robot in 2007 and upgraded to the Xi model in $2017^{10}$. A second surgeon console allows trainee surgeons to operate under direct supervision. In 2009 it was decided to train an RN as a table-side assistant for urology procedures. Since then, four other RNs/NPS have been trained or are in the process of training as table-side assistants in urology. The program of training RNs and NPs as table-side assistants for robotic-assisted procedures was initiated to improve patient safety by enhancing the training of both medical and nursing staff and improving surgical flow and communication in the robot theatre.

An experienced nurse as tableside assistant allows the trainees and registrars to leave the position of table-side assistant and move into the role of supervised console surgeon. While it may be hypothesised that nurses undertaking the table-side robotic assistant role are reducing opportunities for more junior medical personnel to learn, experienced nurses can add value by providing learning opportunities to junior medical staff in the role of table-side assistant while maintaining patient safety and allowing the consultant surgeon to give instruction to more senior trainees as the console surgeon ${ }^{17,18}$. While the trainee is learning the role of console surgeon they are supported by a consultant surgeon on a second console and an experienced nurse as the table-side assistant. The nurse as table-side robotic surgical assistant is a constant in the surgical unit while junior medical personnel work through rotations in various specialties. The nurse as surgical assistant brings consistency and offers a point of coordination and communication to public sector units where registrars change units and consultant surgeons and anaesthetists are often part-time employees. The expert nurse as table-side assistant is a valuable resource for tasks such as positioning, prepping and draping, and troubleshooting technical issues with the robot. The consistency in the role supports efficiencies in the operating room related to turnover, set-up time and supporting nursing staff as they become familiar with the roles of circulating and instrument nurse for robotic-assisted procedures. All of these tasks enhance patient safety and improve surgical flow.

In addition to supporting nursing and medical education, the nurse as surgical assistant adds value to the public health care sector by increasing nurses' job satisfaction, therefore allowing nurses to achieve professional progression while remaining in the clinical setting rather than moving into a management or education role. This would subsequently lead to increased retention (and attraction) of experienced nurses. The extended practice role also has the potential to attract novice nurses to the unit Having an RN or NP as table-side assistant can aid in establishing a cohesive, high-functioning and efficient robotic nursing team which has the potential to attract consultants to the unit and, as a result, increase utilisation of the robot. An RN or NP in an extended practice role can also add value in the pre-operative workup of patients - for example by flagging issues, such as an absence of consent or other documentation, before the day of surgery which has the potential to avoid delays or cancellations of procedures. The role adds continuity of care through the perioperative phases and allows for individual planning for each patient's surgical procedure.

It is predicted that there will be a shortfall in the nursing workforce by 2025 due to the combination of Australian population health care trends, the aging nursing workforce and poor retention rates 
within the nursing profession ${ }^{19,20}$. With this in mind it would seem counterproductive to extend the scope of practice of nurses to incorporate tasks which would traditionally be undertaken by medical staff. However, the literature suggests that workplaces which fail to provide tertiary educated nurses with more complex challenges may lead to employee dissatisfaction which may affect retention ${ }^{21}$. Expanded roles for nurses can assist with workforce retention and provide workforce building opportunities by attracting graduates seeking clinically based career progression ${ }^{21}$. Nurses working in this extended role contribute to the outcomes of the MNHHS Robotic Surgery Plan, which are to facilitate better surgical outcomes for patients, increase workforce knowledge and skills in robotics, and improve job satisfaction ${ }^{10}$.

The nurse as table-side assistant in urology at the RBWH opens opportunities for nursing research into how the extended roles of nurses in the public sector of Australian health care can contribute to efficient and effective surgical care. It also offers the opportunity for other surgical specialties within the RBWH or the wider public health care sector to utilise the experience of perioperative nurses to enhance training of medical and nursing staff.

\section{Educational preparation}

Numerous studies cite the importance of a knowledgeable, cohesive and well-trained robotic team, of which the table-side assistant is a vital member, for achieving a safe and efficient robotic-assisted procedure ${ }^{15}$. The successful completion of roboticassisted surgery is reliant on the proficient and predictive actions of the table-side assistant. Highlighted by Nayyar et al.', undertaking the role

Complies with ACORN statement regarding AHPRA registration and perioperative experience for a nurse surgical assistant ${ }^{1,4}$

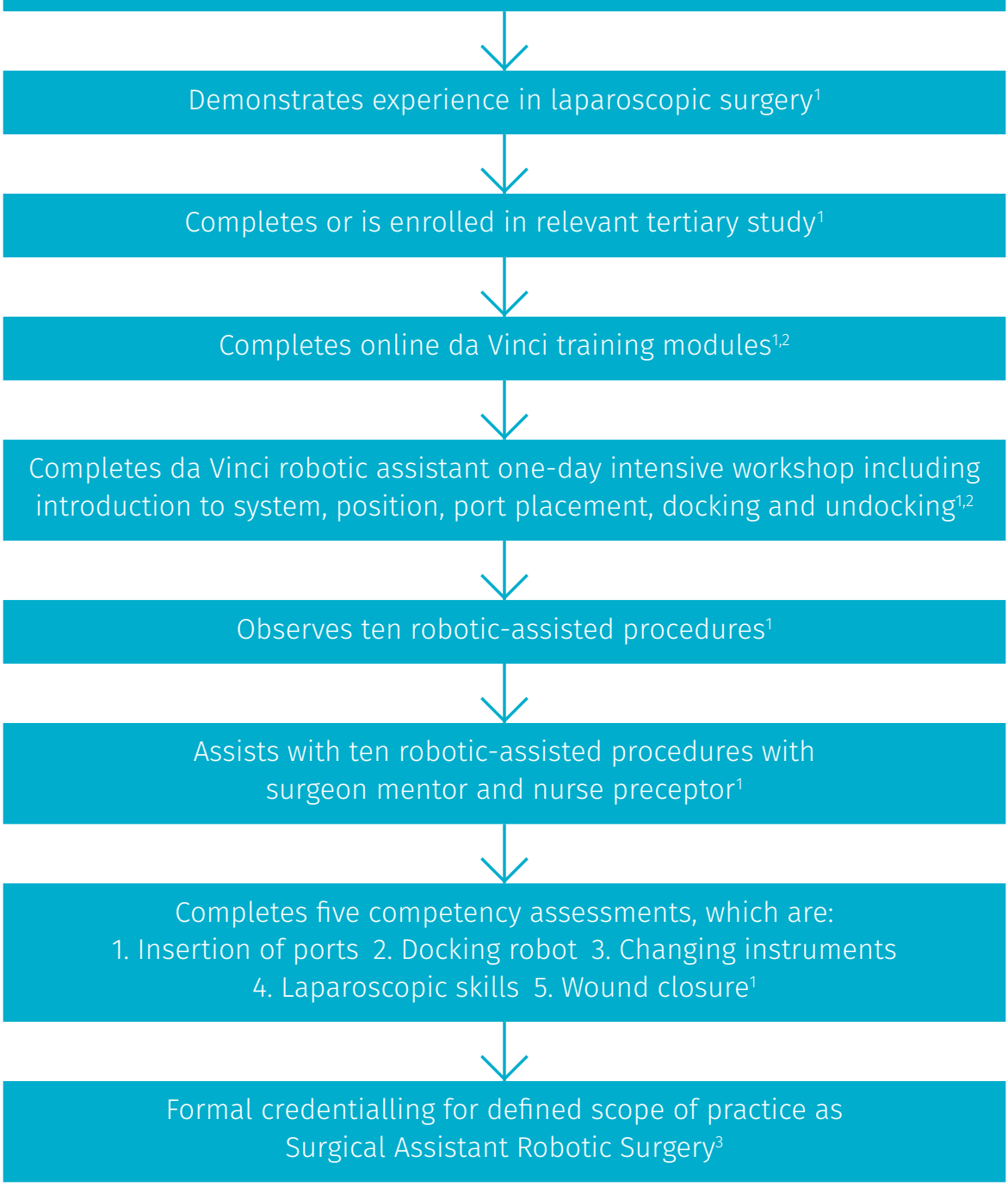

Figure 1: Training and credentialling requirements for registered nurses undertaking the role of assistant during robotic surgery at Royal Brisbane and Women's Hospital

1. Royal Brisbane and Women's Hospital (RBWH). RBWH Procedure 001616: Registered Nurse Surgical Assistant Role Version 3. Brisbane: RBWH, April 2018.

2. Metro North Hospital and Health Service (MNHHS). MNHHS da Vinci Robotic Surgery Reference Guide Version 4.7. Brisbane: MNHHS, August 2019.

3. Metro North Hospital and Health Service (MNHHS). MNHHS Robotic Surgery Plan 2018-2021 Version 4. Brisbane: MNHHS, May 2018.

4. Australian College of Perioperative Nurses (ACORN). Standards for Perioperative Nursing in Australia $16^{\text {th }}$ ed. Adelaide: ACORN, 2020.

of table-side assistant for roboticassisted surgery is a complex task. While the console surgeon has a three-dimensional view of the surgical site in the surgeon console, the assistant has a two-dimensional view utilising only one camera of the telescope which results in a lack of depth perception. The assistant only has access from one side of the patient, which restricts access to some anatomy, and introduction 
of the assistant's instruments are seldom camera-guided. Limited access is compounded by the robotic instruments occupying most of the available surgical space and, because of their freedom of movement, the robotic instruments will often move the assistant's instruments?

Sgarbura ${ }^{16}$ states that table-side assistants with formal training have improved efficiency in tasks such as placing instruments. Thiel et al. ${ }^{15}$ proposed a three-phase table-side assistant training protocol consisting of hands-on training related to the functions of the robot including docking procedures, instrument exchanges and arm manipulation; a review of a customised unit video of the surgical procedure emphasising the anatomy and particularly focusing on the assistants role in each of ten highlighted stages of the (prostatectomy) procedure; and a hands-on simulation component to gain and hone laparoscopic instrument skills. They concluded that not only novice table-side assistants benefited from this type of training but also experienced robotic assistants who expressed the value of ongoing training of this kind ${ }^{15}$.

Nurses training as table-side assistants at the RBWH are mentored by robotic-trained consultants and receive hands-on sessions with the company representative specifically targeted to the functions of the three components of the robot, that is, the patient cart, vision cart and surgeon console. The company also provides comprehensive online learning packages for the console surgeon, table-side assistant and the extended robotic team. The nurse as a novice table-side assistant also receives supervision during live cases from an experienced table-side assistant nurse. RBWH has a comprehensive training and credentialing process for nurses undertaking defined scope of clinical practice which includes the role of surgical assistant robotic surgery (see Figure 1).

\section{Conclusion}

The RN or NP as a table-side robotic assistant has been successfully implemented at the RBWH in the surgical specialty of urology and has the potential to expand across several specialties. The role adds consistency and a point of communication and coordination as medical personnel of all levels rotate through the unit. This consistency and enhanced communication increases patient safety and surgical flow.

The nurse as a table-side assistant adds value by allowing senior training medical staff to acquire skills as the console surgeon and gives junior medical staff the opportunity to learn the role of table-side assistant with instruction from an experienced nurse. This role also promotes the education of nurses in the roles of circulating and instrument nurse. In addition, as it is an extended role the nurse as a table-side assistant offers increased job satisfaction for nursing staff with the potential to attract both experienced and novice nurses to the unit.

\section{References}

1. Hains T, Rowell D, Strand H. The legitimacy of the nurse practitioner as a non-medical surgical assistant: Historical evidence from Australia. Collegian 2020;27:654-660.

2. Australian College of Perioperative Nurses (ACORN). Standards for Perioperative Nursing in Australia 2018. Adelaide, SA; ACORN; 2018.

3. Hains T, Rowell D, Strand H. Effectiveness of the non-medical surgical assistant measured by patient outcome assessment. Int J Nurs Pract 2020:e12822.

4. Hains T, Turner C, Strand H. Complexities of the Australian perioperative nurse entrepreneur. Aust J Adv Nurs 2018;36:1:48-55.
5. Duff J. Will robots make good perioperative nurses? Journal of Perioperative Nursing 2020;33(3):e-1-2.

6. Maric S. Robot instead of a surgeon, reality or a media trick? [Internet]. Sarajevo: WizardHealth; 2016 [cited 2020 September 9]. Available from: www.wizardhealth.co/robotinstead-of-a-surgeon-reality-or-a-mediatrick/?lang=en.

7. Nayyar R, Yadav S, Singh P, Dogra P. Impact of assistant surgeon on outcomes in robotic surgery. Indian J Urol 2016;32(3):204-209.

8. Mangano MS, Lamon C, Beniamin F, De Gobbi A, Ciaccia M, Maccatrozzo L. The role of bedside assistant during robot assisted radical prostatectomy: Is more experience better? Analysis on perioperative and clinical outcomes. Urologia 2020:391560320951085.

9. Perez RE, Schwaitzberg SD. Robotic surgery: Finding value in 2019 and beyond. Ann Laparosc Endosc Surg 2019;4:51.

10. Metro North Hospital and Health Service. Robotic Surgery Plan 2018-2021. Brisbane: Queensland Government; 2018.

11. Yaxley JW, Coughlin GD, Chambers SK, Occhipinti S, Samaratunga H, Zajdlewicz $L$ et al. Robot-assisted laparoscopic prostatectomy versus open radical retropubic prostatectomy: Early outcomes from a randomised controlled phase 3 study. Lancet 2016;388(10049):1057-1066.

12. Potretzke A, Knight B, Brockman J, Vetter J, Figenshau R, Bhayani S et al. The role of the assistant during robot-assisted partial nephrectomy: Does experience matter? J Rob Surg 2016;10(2):129-134.

13. Khene Z, Peyronnet B, Bosquet E, Pradere B, Verhoest G, Mathieu R et al. Analysis of the impact of the assistant surgeon experience on perioperative outcomes of robotic partial nephrectomy. J Urol 2017;197:4S.

14. McCrorey M, Kitahara H, Krienbring D, Patel B, Nisivaco S, Balkhy HH. Robotic cardiac surgery impact of a new patientside assistant on outcomes. Gen Thorac Cardiovasc Surg 2020;68(1):24-29.

15. Thiel DD, Lannen A, Richie E, Dove J, Gajarawala NM, Igel TC. Simulation-based training for bedside assistants can benefit experienced robotic prostatectomy teams. J Endourol 2012;27(2):230-237.

16. Sgarbura $O$, Vasilescu $C$. The decisive role of the patient-side surgeon in robotic surgery. Surg Endosc 2010;24(12):3149-3155.

17. Quick J. The role of the surgical care practitioner within the surgical team. $\mathrm{Br}$ Nurs 2013;22(13):759-765.

18. Quick J, Hall S. Non-medical surgical assistants: A review. Clin Serv J 2014; June:75-78. 
19. Health Workforce Australia. Australia's Future Health Workforce - Nurses Detailed Report. Canberra: Department of Health; 2014.

20. Health Workforce Australia. Nursing Workforce Sustainability: Improving Nurse Retention and Productivity. Canberra: Department of Health; 2014.

21. Duckett S. Health workforce design for the $21^{\text {st }}$ century. Australian Health Review. 2005;29(2):201-210. 